\title{
Patients' attitudes towards mobile health in Singapore: a cross-sectional study
}

\author{
Ihtimam Hossain ${ }^{1}$, Ying Na Ang ${ }^{2}$, Hui Ting $\mathrm{Chng}^{2}$, Pei Shieen Wong ${ }^{1,2}$ \\ ${ }^{1}$ Department of Pharmacy, Singapore General Hospital, Singapore; ${ }^{2}$ Department of Pharmacy, National University of Singapore, Singapore \\ Contributions: (I) Conception and design: All authors; (II) Administrative support: I Hossain, HT Chng, PS Wong; (III) Provision of study materials \\ or patients: I Hossain, PS Wong; (IV) Collection and assembly of data: I Hossain, YN Ang, PS Wong; (V) Data analysis and interpretation: All \\ authors; (VI) Manuscript writing: All authors; (VII) Final approval of manuscript: All authors. \\ Correspondence to: Pei Shieen Wong; Ihtimam Hossain. Department of Pharmacy, Singapore General Hospital, Outram Road, 169608, Singapore. \\ Email: wong.pei.shieen@sgh.com.sg; ihtimam.hossain@gmail.com.
}

\begin{abstract}
Background: Smartphone-mediated mobile health (mHealth) has the potential to assist patients with medication adherence and disease monitoring. This study aimed to describe the awareness and usage of, and attitudes towards, mHealth among smartphone-owning patients in a tertiary hospital in Singapore.

Methods: A self-administered cross-sectional survey was systematically offered to patients at the Singapore General Hospital from August to September 2018. Participants were included if they were at least 18 years old, owned a smartphone, and could speak and read simple English. No identifiable data was collected. Responses were summarized using descriptive statistics. Multiple logistic regression analysis was used to identify factors associated with awareness and usage of, and attitudes towards, mHealth.

Results: Four-hundred and two eligible responses were received, with most participants reporting having completed tertiary education (63.7\%) and having chronic medical conditions (71.1\%), with a mean age of about 43 years. On average, participants were aware of 3.7 out of $7 \mathrm{mHealth}$ functions and used 1.9 functions. Most patients were aware that smartphones could be used for general health/fitness tracking, obtaining health information, and appointment management. Most (76.3\%) participants were keen to learn to use mHealth in future, and $63.2 \%$ agreed that mHealth could help them better manage their health.

Conclusions: Although mHealth usage among patients was low, most patients held positive attitudes towards mHealth. For mHealth to fulfill its potential, strategies to improve the awareness and usage among patients need to be explored.
\end{abstract}

Keywords: Mobile health; Singapore; smartphone; health knowledge, attitudes, practice

Received: 12 May 2019; Accepted: 08 August 2019; Published: 16 September 2019.

doi: $10.21037 /$ mhealth.2019.08.07

View this article at: http://dx.doi.org/10.21037/mhealth.2019.08.07

\section{Introduction}

Mobile health (mHealth) refers to medical and public health practice supported by mobile devices, such as mobile phones, patient monitoring devices, personal digital assistants, and other wireless devices (1). Although a relatively new concept, some studies have demonstrated that mHealth helps to improve the management of chronic diseases such as diabetes, hypertension and asthma through lifestyle management, medication adherence and engagement in self-care (2-7). Mobile health solutions, which could be as simple as automated text message reminders, are potentially low cost and scalable (8-10). Although improvements in long-term clinical outcomes have yet to be clearly demonstrated, the evidence supports that mHealth can empower patients and potentially improve health outcomes (11).

Singapore is a multicultural city state in South East 
Asia (population: 5.6 million) with an efficient and technology-driven public healthcare system (12-14). It has one of the highest mobile phone penetrations (147.3\% in August 2018) in the world, with an estimated $76 \%$ of the population using smartphones $(15,16)$. According to the World Health Organization's $3^{\text {rd }}$ Global Survey on eHealth, most mHealth program types were already established in Singapore (17). Furthermore, Singapore, like many developed countries, is facing the impending challenges of an ageing population with an increasing prevalence of chronic diseases (18). Therefore, effective implementation of mHealth programs could assist in tackling some of these challenges proactively through active prevention and care integration.

Apart from the technical prerequisites, cultural practices and attitudes influence the successful adoption and implementation of new technologies and innovations. In Singapore, previous studies have been done regarding specific app-based interventions or among defined patient groups, for example type 2 diabetes mellitus patients, coronary heart disease patients, and patients on oral anticancer medications (19-22). Little is known about the usage patterns and attitudes towards smartphone-mediated mHealth among patients in general. For example, it is difficult to find reliable estimates for the proportion of patients who are aware of mHealth interventions and use them.

A previous study by our research team identified generally positive attitudes towards mHealth amongst the general population, albeit with relatively low mHealth usage (as might be expected of generally young and healthy participants) (23). Managing appointments, and fitness and diet tracking were the most popular mHealth functions in terms of both awareness and usage. That said, the awareness and usage patterns among patients are expected to be different. Furthermore, as patients are the users who might benefit the most from mHealth, it is important to understand their attitudes towards mHealth.

This study aims to describe current knowledge, practices and attitudes towards mHealth among patients at a tertiary hospital in Singapore who own a smartphone. It also attempts to identify factors that influence the above in the study population. Acknowledging and understanding these attitudes and behaviors will allow future mHealth solutions to be optimized to realize the benefits of mHealth in Singapore and similar societies.

\section{Methods}

\section{Study design}

The study was a cross-sectional survey of patients who visited the outpatient and discharge pharmacies in Singapore General Hospital (SGH), across a 3-week period in August and September 2018. Patients who were 18 years and above, owned a smartphone and able to speak and read English were eligible for the study. Patients were systematically offered participation in this study, according to their queue number.

After obtaining verbal informed consent, a selfadministered questionnaire was handed to the participant. Recruitment continued until a target of 400 eligible responses were received. As participation in the study was voluntary and no identifiable information was collected, exemption from full Institutional Review Board review was granted.

\section{Survey development \& pretesting}

The survey instrument was modified from the one used in a prior study assessing public attitudes towards mHealth (23). The survey instrument included multiple choices, modified Likert scales, and yes/no questions. The survey was originally developed based on tools used in similar crosssectional studies pertaining to mHealth, though discretion was exercised to ensure relevance to a Singapore context. mHealth functions were grouped broadly into 7 categories, which were adapted from categories used in the WHO's $3^{\text {rd }}$ Global Survey on eHealth: managing appointments, accessing health records, health information/education, general health and fitness tracking, disease monitoring, medication management, and contacting healthcare professionals.

Survey questions were organized into 4 sections: (I) participant demographics, and background information; (II) baseline general smartphone usage; (III) awareness and usage of mHealth functions; and (IV) attitudes towards mHealth in general, and factors influencing receptivity towards mHealth apps. Simplicity was a guiding principle, to ensure that participants with a basic understanding of written English (equivalent to an elementary level) would be able to understand and complete the survey in 10 minutes with minimal assistance.

The proposed survey instrument was pretested with 
fifty participants (including middle-aged and elderly participants). Modifications were made based on feedback to improve ease of understanding. The final survey took the form of a 6-page printed questionnaire.

\section{Participants \& survey administration}

Patients presenting to both inpatient (discharge) and outpatient pharmacies (i.e., collecting medications for themselves) were systematically sampled based on their queue numbers. The study objectives and risks were explained to potential participants by a study team member. Participants who agreed were then handed a printed questionnaire and were encouraged to submit the completed questionnaire into a drop-box before leaving the pharmacy. The target recruitment was set at 400 completed questionnaires, with about $10 \%$ from the discharge pharmacy (proportionate to the number of patients discharged relative to number of specialist outpatient attendances to the hospital).

\section{Statistical analysis}

Descriptive statistics for categorical data were reported using frequencies and percentages. Subgroup analyses were conducted based on responses to section (I) of the survey (demographics and background smartphone usage), to assess whether they had an influence on responses on sections (II) to (IV) of the survey (awareness and usage of, and attitudes towards, mHealth). Responses for sections (II), (III), and (IV) were converted to numerical scores for each participant, and their means were compared between subgroups. For example, mHealth awareness was scored based on the number of "yes" responses across the 7 categories to derive a score out of 7 .

Univariate dichotomous subgroup analyses were conducted using the Mann-Whitney $U$ test to compare mean scores across responses, and Pearson's chisquared test to compare proportions. Factors identified as statistically significant were then compared using a multiple logistic regression to identify the factors that were independently associated with the outcomes after adjustment. All tests were two-sided and a $\mathrm{P}$ value of $<0.05$ was considered statistically significant. As subgroup analyses were considered hypothesis-generating, no adjustments were made for multiple comparisons. Data analyses were performed with SPSS Statistics Version 25.0 for Mac OS (SPSS Inc., Chicago, IL, USA).

\section{Results}

\section{Participant characteristics}

Six-hundred and ninety-two patients were approached, of whom 402 participants met the inclusion criteria and agreed to participate. The mean age of patients was 42.6 years old. Majority of them were Chinese, completed tertiary education, employed and lived in public housing. Most patients reported having at least 1 chronic medical condition $(71.1 \%)$ and were taking regular medications (74.1\%). Only a minority (3.2\%) reported having at least 4 chronic conditions, and about a third of the patients reported having been hospitalized in the past year. About a third of the patients reported taking health supplements regularly, and only a minority reported taking traditional medicines (in this context, referring to forms of alternative or complementary medicine such as traditional Chinese herbal medicine, and acupuncture) regularly (Table 1).

Only patients who owned smartphones were recruited to participate in the study. Of the participants, all reported being able to independently make calls or use short message service (SMS). On average, each participant reported using 6.7 out of 8 smartphone functions listed in the survey (i.e., smartphone usage score) (Table 2). More than half of the patients $(62.4 \%)$ used $\geq 7$ of the 8 smartphone functions listed in the questionnaire.

The most popular sources from which patients reported obtaining health information were 'website/internet/online sources' (72.9\%), 'Newspaper, magazines and other printed materials' (51.0\%) and 'Healthcare providers' (49.5\%).

\section{General mHealth awareness, usage, and attitudes}

General health and fitness tracking (83.1\%), health information and education (78.4\%), and managing appointments $(75.1 \%)$ were the mHealth functions with the highest awareness among participants. A minority of patients were aware of disease monitoring (34.6\%) and medication management $(23.4 \%)$ as mHealth functions. The mean (SD) mHealth awareness score (out of 7) was 3.7 (1.9) (Table 3).

Likewise, with mHealth usage, health information and education, general health and fitness tracking, and managing appointments (41.0\%), were the most used functions. Only a minority of participants reported using smartphones for disease monitoring and medication management. The mean (SD) mHealth usage score (out of 7) was 1.9 (1.6) (Table 3).

All of the participants were asked whether they felt 
Table 1 Baseline demographic \& medical characteristics of participants

\begin{tabular}{|c|c|}
\hline Characteristics & Respondents $(\%)^{\dagger}(\mathrm{n}=402)$ \\
\hline \multicolumn{2}{|l|}{ Demographic } \\
\hline \multicolumn{2}{|l|}{ Survey location } \\
\hline Outpatient pharmacies & $362(90.0)$ \\
\hline Discharge pharmacy & $40(10.0)$ \\
\hline \multicolumn{2}{|l|}{ Sex } \\
\hline Male & $193(48.0)$ \\
\hline Female & $209(52.0)$ \\
\hline Age (years), mean $\pm S D$ & $42.6 \pm 13.0$ \\
\hline \multicolumn{2}{|l|}{ Ethnicity } \\
\hline Chinese & $255(63.4)$ \\
\hline Malay & $72(17.9)$ \\
\hline Indian & $51(12.7)$ \\
\hline Others, or not reported & $24(6.0)$ \\
\hline \multicolumn{2}{|l|}{ Languages (spoken) } \\
\hline English & $402(100.0)$ \\
\hline Mandarin & $252(62.7)$ \\
\hline Malay & $51(12.7)$ \\
\hline Tamil & $31(7.7)$ \\
\hline \multicolumn{2}{|l|}{ Marital status } \\
\hline Single & $138(34.3)$ \\
\hline Married & $246(61.2)$ \\
\hline Divorced & $9(2.2)$ \\
\hline Widowed & $9(2.2)$ \\
\hline \multicolumn{2}{|l|}{ Employment status } \\
\hline Employed (full-time) & $315(78.4)$ \\
\hline Employed (part-time) & $21(5.2)$ \\
\hline Unemployed/retired & $66(16.4)$ \\
\hline \multicolumn{2}{|l|}{ Educational level } \\
\hline Degree/diploma & $256(63.7)$ \\
\hline Secondary or pre-university & $123(30.6)$ \\
\hline Primary or below & $23(5.7)$ \\
\hline \multicolumn{2}{|l|}{ Residential status } \\
\hline Public housing & $338(84.1)$ \\
\hline Private housing & $64(15.9)$ \\
\hline
\end{tabular}

Table 1 (continued)
Table 1 (continued)

\begin{tabular}{lc}
\hline Characteristics & Respondents $(\%)^{\dagger}(\mathrm{n}=402)$ \\
\hline Medical & \\
Chronic conditions & $116(28.9)$ \\
None & $273(67.9)$ \\
1 to 3 & $13(3.2)$ \\
4 or more & \\
Hospitalizations (in the past year) & $265(65.9)$ \\
None & $115(28.6)$ \\
1 to 2 & $22(5.5)$ \\
3 or more
\end{tabular}

Chronic disease visits (per year), $\mathrm{n}=286^{\ddagger}$

Up to 2

$118(41.3)$

3 to 4

$128(44.8)$

5 or more

$40(14.0)$

Regular medications (daily)

None

104 (25.9)

1 to 3

222 (55.2)

4 or more

$76(18.9)$

Compliance, $\mathrm{n}=298^{\S}$

Always/usually

$281(94.3)$

Sometimes/rarely

$17(5.7)$

Others (regular)

Traditional medicines use

$41(10.2)$

Health supplements use

$143(35.6)$

${ }^{\dagger}$, unless otherwise specified; ${ }^{\ddagger}$, only including participants reporting chronic conditions; ${ }^{\S}$, only among participants who reported taking regular medications.

each of the seven functions were useful, and these were then converted to 'usefulness' scores (out of 1) to allow for comparison (very useful: 1 point; somewhat useful: 0.5 point; not useful at all: 0 points). Managing appointments had the highest overall usefulness score $(0.81)$, followed by health information and education (0.75), and general health and fitness tracking (0.73). Medication management had the lowest usefulness score (0.63), with only $35.1 \%$ of respondents considering it to be very useful. On average, the mean mHealth usefulness score across all seven 
functions was 0.70 (Table 4).

Participants were asked to rate how much they agreed or disagreed with a series of five statements to assess some of their attitudes towards mHealth. Most of the patients were keen to learn and try mHealth in the future $(73.6 \%)$. In addition, over half of patients agreed that mHealth could help them manage their health better $(63.2 \%)$ and improve communication with healthcare providers (62.4\%). Similarly, $56.5 \%$ of patients were keen to share their health data with their family members and $60.7 \%$ were keen to do so with their healthcare providers (Table 5).

Table 2 Baseline smartphone usage characteristics of participants

\begin{tabular}{lc}
\hline Baseline smartphone usage & Respondents (\%) (n=402) \\
\hline Smartphone use & $359(89.3)$ \\
Sending \& receiving E-mails & $378(94.0)$ \\
Alarms \& time management & $369(91.8)$ \\
Entertainment & $389(96.8)$ \\
Social media/communication apps & $300(74.6)$ \\
Reading news & $248(61.7)$ \\
Online shopping/ordering & $350(87.1)$ \\
Transport \& navigation & $278(69.2)$ \\
Banking \& electronic payments & $6.7 \pm 1.6$ \\
Use score (out of 8), mean \pm SD &
\end{tabular}

The "smartphone use score" is a count of the number of functions (out of the 8 surveyed) a respondent regularly (and independently) uses his or her smartphone for. Also, all participants surveyed reported being able to make calls or send text messages independently.
Participants were also asked to rate how important they felt six features were in influencing their receptivity to using mHealth. Majority of patients felt that data security and privacy $(89.6 \%)$, reliability $(60.9 \%)$, and being easy to learn $(83.1 \%)$ were very important. Only a minority of participants felt that way about automatic login (36.3\%), which referred to the absence of further authentication at the point of starting an application (such as reentry of passwords, pins, or fingerprint recognition), excluding the initial set-up (Table 6).

\section{Factors influencing mHealth awareness, usage, and attitudes}

Univariate analyses suggested that higher mHealth awareness scores (out of 7) were associated with age below 50 years (3.92 vs. 3.20, $\mathrm{P}<0.001)$, having completed tertiary education (3.88 vs. 3.32, $\mathrm{P}=0.006)$, and having a smartphone use score $\geq 7$ (4.05 vs. 3.59, $\mathrm{P}<0.001$ ). After adjustment using binary logistic regression, only a higher smartphone use score was significantly associated with an awareness score of at least 4 (OR: $1.43, \mathrm{P}<0.001)$. Age and education were no longer statistically significant (Table 7).

Univariate analyses suggested that higher mHealth usage scores (out of 7) were associated with age below 50 years (2.13 vs. 1.38, $\mathrm{P}<0.001)$, being employed (1.93 vs. 1.56 , $\mathrm{P}=0.018)$, having completed tertiary education (2.20 vs. $1.29, \mathrm{P}<0.001)$, and having a smartphone use score $\geq 7$ (2.26 vs. $1.21, \mathrm{P}<0.001)$. After adjustment using binary logistic regression, only a higher smartphone use score (OR: 1.73, $\mathrm{P}<0.001)$, was significantly associated with a mHealth usage score of at least 2 (Table 7).

Table 3 Mobile health awareness and usage among participants $(n=402)$

\begin{tabular}{lcc}
\hline Mobile health functions & Awareness, $n(\%)^{\dagger}$ & $U_{\text {sage, } \mathrm{n}(\%)^{\ddagger}{ }^{\dagger}}$ \\
\hline Managing appointments & $302(75.1)$ & $57(34.3)$ \\
Accessing health records & $166(41.3)$ & $243(77.1)$ \\
Health information or education & $315(78.4)$ & $169(50.6)$ \\
General health and fitness tracking & $334(83.1)$ & $47(33.8)$ \\
Disease monitoring & $139(34.6)$ & $22(23.4)$ \\
Medication management & $94(23.4)$ & $48(37.8)$ \\
Contacting or consulting healthcare providers & $127(31.6)$ & $1.9 \pm 1.6$ \\
\hline
\end{tabular}

${ }^{\dagger}$, unless otherwise specified; ${ }^{\ddagger}$, usage percentage reported as proportion of participants who were aware of that specific mHealth function.

Respondents who declared being unaware of a specific function were defaulted to 'non-users'. 
Table 4 Participants' perceived usefulness of mobile health

\begin{tabular}{|c|c|c|c|c|}
\hline Mobile health attitudes & Not useful at all ${ }^{\dagger}$ & Somewhat useful $\left.\right|^{\dagger}$ & Very useful ${ }^{\dagger}$ & Usefulness score ${ }^{\ddagger}$ \\
\hline Accessing health records & $42(10.4)$ & $161(40.0)$ & 199 (49.5) & 0.70 \\
\hline Health information and education & $15(3.7)$ & $168(41.8)$ & $219(54.5)$ & 0.75 \\
\hline General health and fitness tracking & $14(3.5)$ & $188(46.8)$ & $200(49.7)$ & 0.73 \\
\hline Medication management & $33(8.2)$ & $228(56.7)$ & $141(35.1)$ & 0.63 \\
\hline Contacting healthcare providers & $33(8.2)$ & $185(46.0)$ & $184(45.8)$ & 0.69 \\
\hline Mean $\pm \mathrm{SD}^{\ddagger}$ & & & & $0.71 \pm 0.06$ \\
\hline
\end{tabular}

${ }^{\dagger}$, reported as $\mathrm{n}(\%) ;{ }^{\ddagger}$, the 'usefulness score' is a weighted average computed by assigning scores of $0,0.5$, or 1 to the possible responses ("not useful at all", "somewhat useful", and "very useful" respectively). A higher score implies a more favourable attitude towards the usefulness of the specific mHealth function. The mean usefulness score of all the functions is a measure for participants' attitude towards usefulness of $\mathrm{mHealth}$ in general.

Table 5 Participants' attitudes towards mobile health

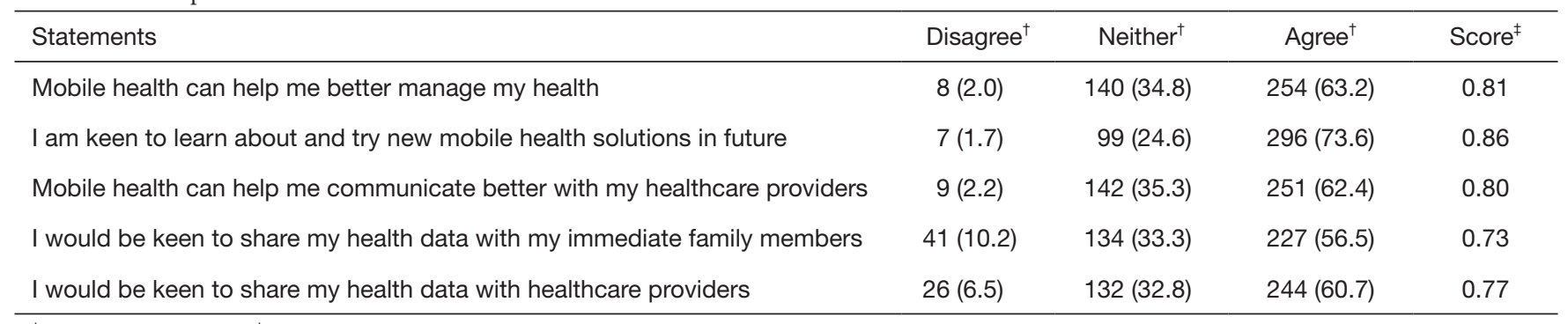

${ }^{\dagger}$, reported as $\mathrm{n}(\%) ;{ }^{\ddagger}$, this score is a weighted average computed by assigning scores of $0,0.5$, or 1 to the possible responses ("disagree",

"neither agree nor disagree", and "agree" respectively). A higher score implies a higher level of agreement with the statement.

Table 6 Factors influencing participants' receptiveness to using mobile health solutions

\begin{tabular}{|c|c|c|c|c|}
\hline Factors & Not important at all ${ }^{\dagger}$ & Somewhat important $^{\dagger}$ & Very important $^{\dagger}$ & Importance score f $^{\ddagger}$ \\
\hline Simple interface & $6(1.5)$ & $75(18.7)$ & 321 (79.9) & 0.89 \\
\hline Easy to learn & $3(0.7)$ & $65(16.2)$ & $334(83.1)$ & 0.91 \\
\hline Multiple language support & $26(6.5)$ & $130(32.3)$ & $246(61.2)$ & 0.77 \\
\hline Rewards for using & $108(26.9)$ & $178(44.3)$ & $116(28.9)$ & 0.51 \\
\hline Automatic login & $86(21.4)$ & $170(42.3)$ & $146(36.3)$ & 0.57 \\
\hline Reliability & $4(1.0)$ & $53(13.2)$ & $245(60.9)$ & 0.92 \\
\hline
\end{tabular}

${ }^{\dagger}$, reported as $\mathrm{n}(\%) ;{ }^{\ddagger}$, the 'importance score' is a weighted average computed by assigning scores of $0,0.5$, or 1 to the possible responses ("not important at all", "somewhat important", and "very important" respectively). A higher score implies more importance ascribed to that factor by respondents collectively. 
Table 7 Logistic regression analysis of factors influencing awareness, usage, and attitudes towards mHealth

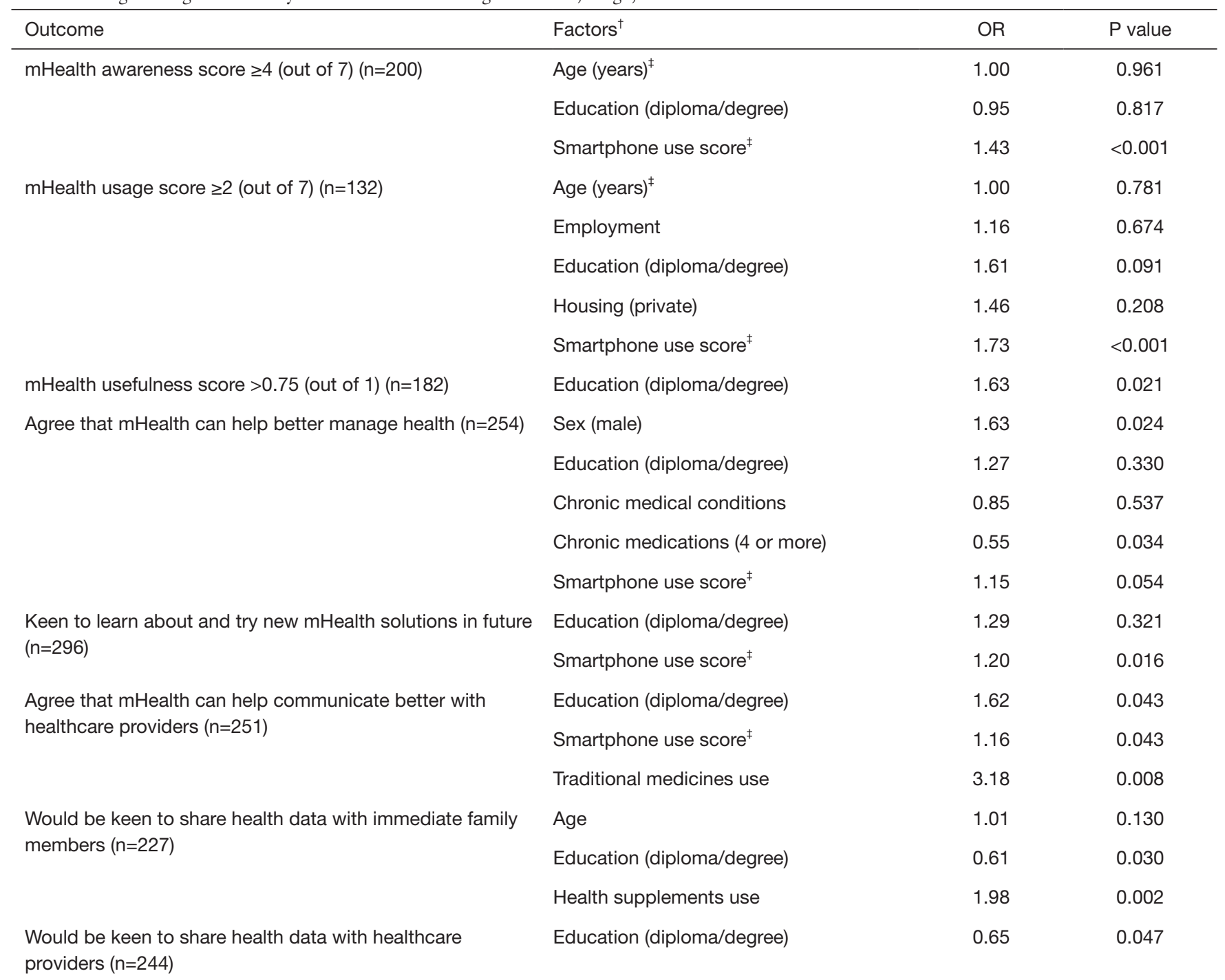

${ }^{\dagger}$, only factors identified as statistically significant in univariate analysis were included in the multiple logistic regression models. Subsequently, $\mathrm{P}$ values $<0.05$ were considered statistically significant (factors in bold). Odds-ratio (OR) $>1$ implies a positive relationship with the outcome, OR $<1$ implies a negative relationship with the outcome; ${ }^{\ddagger}$, for age and smartphone use score, the ORs are in relation to a one unit increase in the factor (i.e., a 1 year increase in age, or a 1 unit increase in smartphone use score).

After adjustment, male participants were more likely to agree that mHealth could help them better manage their health (OR: $1.63, \mathrm{P}=0.024$ ), but participants with 4 or more chronic medications per day were less likely to agree (OR: 0.55, $\mathrm{P}=0.034)$. Participants with higher smartphone use scores (OR: 1.20, $\mathrm{P}=0.016$ ), were more likely to be receptive to learning or trying mHealth in future. Users of traditional medicines, participants with tertiary education, and those with higher smartphone use scores were all more likely to agree that mHealth could help them communicate better with healthcare providers. Participants with tertiary education were more likely to assign higher usefulness scores to mHealth, but less likely to be keen to share their health data with family members and healthcare providers. Users of health supplement were more likely to be keen to share their health data with their family members (Table 7).

\section{Discussion}

The systematic sampling process obtained a diverse sample of smartphone-owning patients at a tertiary hospital in Singapore. The sample included roughly equal proportions 
of male and female patients (with median \& mean age of about 42 years), and a representative proportion of ethnic minorities in Singapore. The participants used a mean of 6.7 out of 8 smartphone functions (median 7), suggesting a generally high baseline familiarity with smartphones.

The participants reported relatively low awareness (mean 3.7 of 7 functions) and usage (mean 1.9 of 7 functions) of mHealth. In our earlier study among the public, these figures were 4.4 and 2.2, respectively. Most patients were aware of appointment management, general health and fitness tracking, and health information/education functions. Using smartphones to access health information was the most used function (about $60 \%$ of all patients reported doing so). This suggests patients are generally comfortable seeking health information online today although previous studies have highlighted some barriers to this $(24,25)$.

On the other hand, the use of smartphones for disease monitoring, contacting or consulting healthcare providers (e.g., telemedicine, tele-pharmacy etc.), and especially medication management had very low usage. Among those who were aware of those mHealth functions, only between a quarter to a third of them reported using them. This suggests a disconnect between the intentions of tool creators, and actual usage by patients for whom such tools are ostensibly designed. This observation could also reflect the lack of integration of mHealth into routine care by healthcare professionals. A recent study among elderly patients with cardiovascular diseases (CVD) in Singapore identified usability concerns and perceived lack of need as barriers to acceptance of mHealth medication adherence tools (22). Similar barriers were reported among patients on oral chemotherapy (21).

On top of barriers to usage, our results identify a clear awareness gap among patients (regardless of age) about such apps. Only about a third of the patients were aware of disease monitoring, and less than a quarter were aware of medication management. Given that poor adherence or disease management is a common problem among patients with chronic diseases, we believe that there is a need to increase awareness of mHealth among these patients through various channels. Healthcare professionals especially have an important role to play in understanding the landscape and advising their patients on which mHealth solutions might be suitable for them.

Of note, patients with a least 4 chronic medications were less likely to agree that mHealth had the potential to help them better manage their health. Although well-designed studies have demonstrated some benefits of mHealth in short-term outcomes, improvements in long-term clinically important outcomes are still lacking $(11,26,27)$. It is imperative for the digital health industry to find ways to add and demonstrate real value to patients. Further research on patients with relatively complex medical issues may be necessary in this regard.

We attempted to identify factors that influenced awareness and usage of mHealth. However, we did not find any demographic factors that influenced either per se. Only their baseline smartphone use behaviors were independently associated with both - other demographic factors (education and age, in particular) were presumably covariates. Our findings agree with a study conducted among patients with CVD in China, which concluded that information technology skill was the only factor independently influencing willingness to use mHealth (28).

Participants with tertiary education were more likely to perceive mHealth as useful. An exploratory analysis of our results suggests that participants who rated mHealth as more useful were associated with higher mHealth awareness and usage. According to the technology acceptance model, perceived usefulness contributes to intention to use, and thus influences actual use (29). As such, our cross-sectional study design does not allow for exploring this relationship beyond a superficial level. A study specifically looking at mHealth users and how their attitudes have changed before and after using mHealth could shed more light on this.

In this current climate where technology corporations have access to vast amounts of personal information, and recent high-profile cases of health data security breaches (including in Singapore), it is perhaps unsurprising that data privacy and security was rated as very important in influencing receptiveness towards mHealth by most patients (about 90\%). This concern is a widely acknowledged one and requires collaborative solutions between healthcare providers, regulatory authorities, and the industry (30). The Singapore Ministry of Health Licensing Experimentation and Adaptation Programme, a regulatory sandbox, is one such example of a collaborative approach that aims to ensure the risks of mHealth (starting with telemedicine) are mitigated while still allowing for innovative care models to evolve (31).

Despite the low awareness and usage (coupled with low perceived usefulness of some mHealth functions), we identified some positive attitudes towards mHealth. About $63 \%$ of patients believed mHealth could help them manage their health better and improve communication 
with their healthcare providers. Furthermore, almost three quarters of the patients reported being keen to learn about and try mHealth solutions in future. We are hopeful that despite the barriers, with collaborative evidence-based mHealth development, innovative marketing, and effective regulation, the potential benefits of mHealth can be realized.

This study has several key limitations. Firstly, as the questionnaire was self-administered, the responses would be affected by differences in patients' interpretations, and there was no means to verify whether their answers were accurate. Secondly, the survey instrument used has not been independently validated. Finally, the results may not be generalizable to patients in the primary care setting, or to non-English speaking patients.

\section{Conclusions}

In conclusion, this cross-sectional survey reported on the awareness and usage of mHealth among smartphoneowning patients in a tertiary hospital in Singapore. Patients had relatively low awareness and usage of mHealth, particularly regarding disease monitoring and medication management. However, patients held some positive attitudes towards the potential of mHealth, suggesting an opportunity exists to improve usage by increasing awareness and demonstrating the usefulness of mHealth in Singapore.

\section{Acknowledgments}

Funding: This work was supported by the National University of Singapore Pharmacy Final Year Project Grant (C148-000-003-001).

\section{Footnote}

Conflicts of Interest: The authors have no conflicts of interest to declare.

Ethical Statement: As participation in the study was voluntary and no identifiable information was collected, exemption from full Institutional Review Board review was granted (CIRB Ref: 2017/2906). Informed consent was obtained by the study investigators for all participants. The authors are accountable for all aspects of the work in ensuring that questions related to the accuracy or integrity of any part of the work are appropriately investigated and resolved.

\section{References}

1. World Health Organization. mHealth: New horizons for health through mobile technologies: second global survey on eHealth. Available online: https://www.who.int/goe/ publications/goe_mhealth_web.pdf. Published: June 7, 2011. Accessed: September 30, 2018.

2. Curran K, Nichols E, Xie E, et al. An intensive insulinotherapy mobile phone application built on artificial intelligence techniques. J Diabetes Sci Technol 2010;4:209-20.

3. Torbjørnsen A, Jenum A, Småstuen M, et al. A LowIntensity Mobile Health Intervention With and Without Health Counseling for Persons With Type 2 Diabetes, Part 1: Baseline and Short-Term Results From a Randomized Controlled Trial in the Norwegian Part of RENEWING HEALTH. JMIR Mhealth UHealth 2014;2:e52.

4. Piette JD, Datwani H, Gaudioso S, et al. Hypertension management using mobile technology and home blood pressure monitoring: results of a randomized trial in two low/middle-income countries. Telemed J E Health 2012;18:613-20.

5. Logan AG, Irvine MJ, McIsaac WJ, et al. Effect of Home Blood Pressure Telemonitoring With Self-Care Support on Uncontrolled Systolic Hypertension in Diabetics. Hypertension 2012;60:51-7.

6. Liu WT, Huang CD, Wang CH, et al. A mobile telephone-based interactive self-care system improves asthma control. Eur Respir J 2011;37:310-7.

7. Zairina E, Abramson M, McDonald C, et al. Telehealth to improve asthma control in pregnancy: A randomized controlled trial. Respirology 2016;21:867-74.

8. Abdulrahman SA, Rampal L, Ibrahim F, et al. Mobile phone reminders and peer counseling improve adherence and treatment outcomes of patients on ART in Malaysia: A randomized clinical trial. PLoS One 2017;12:e0177698.

9. Arora S, Peters AL, Burner E, et al. Trial to examine text message-based mHealth in emergency department patients with diabetes (TExT-MED): a randomized controlled trial. Ann Emerg Med 2014;63:745-54.e6.

10. Thakkar J, Kurup R, Laba TL, et al. Mobile Telephone Text Messaging for Medication Adherence in Chronic Disease: A Meta-analysis. JAMA Intern Med 2016;176:340-9.

11. Yang Q, Van Stee SK. The Comparative Effectiveness of Mobile Phone Interventions in Improving Health Outcomes: Meta-Analytic Review. JMIR Mhealth Uhealth 
2019;7:e11244.

12. Singapore Department of Statistics. Population Trends, 2018. Available online: http://www.singstat.gov.sg/. Published: September 27, 2018. Accessed: January 19, 2018.

13. Ahmed S, Hasan MZ, MacLennan M, et al. Measuring the efficiency of health systems in Asia: a data envelopment analysis. BMJ Open 2019;9:e022155.

14. Ramesh M, Bali AS. The healthcare system in Singapore. Available online: https://lkyspp.nus.edu.sg/gia/article/thehealthcare-system-in-singapore. Published: May 15, 2017. Accessed: January 22, 2019.

15. Info-communications Media Development Authority. Mobile Penetration Rate. Available online: https:// data.gov.sg/dataset/mobile-penetration-rate. Updated: November 28, 2018. Accessed: Dec 11, 2018.

16. Statista. Smartphone penetration rate as share of the population in Singapore from 2017 to 2023. Available online: https://www.statista.com/statistics/625441/ smartphone-user-penetration-in-singapore/. Published: February 2019. Accessed: March 12, 2019.

17. World Health Organization. Atlas of eHealth country profiles 2015: The use of eHealth in support of universal health coverage. Available online: http://www.who.int/goe/ publications/atlas_2015/en/. Published: February 2016. Accessed: April 30, 2018.

18. Lim WS, Wong SF, Leong I, et al. Forging a FrailtyReady Healthcare System to Meet Population Ageing. Int J Environ Res Public Health 2017;14. doi: 10.3390/ ijerph 14121448 .

19. Goh G, Tan NC, Malhotra R, et al. Short-term trajectories of use of a caloric-monitoring mobile phone app among patients with type 2 diabetes mellitus in a primary care setting. J Med Internet Res 2015;17:e33.

20. Zhang H, Jiang Y, Nguyen HD, et al. The effect of a smartphone-based coronary heart disease prevention (SBCHDP) programme on awareness and knowledge of CHD, stress, and cardiac-related lifestyle behaviours among the working population in Singapore: a pilot randomised controlled trial. Health Qual Life Outcomes 2017;15:49.

21. Ali EE, Chan SSL, Leow JL, et al. User acceptance of an app-based adherence intervention: Perspectives from patients taking oral anticancer medications. J Oncol Pharm Pract 2019;25:390-7.

22. Haldane V, Tan YG, Teo KWQ, et al. Perspectives on Acceptance and Use of a Mobile Health Intervention for the Prevention of Atherosclerotic Cardiovascular Disease in Singapore: Mixed-Methods Study. JMIR Mhealth Uhealth 2019;7:e11108.

23. Hossain I, Lim ZZ, Ng JJL, et al. Public attitudes towards mobile health in Singapore: a cross-sectional study. Mhealth 2018;4:41.

24. Chang L, Chiuan Yen C, Xue L, et al. Factors associated with mobile health information seeking among Singaporean women. J Women Aging 2017;29:75-86.

25. Chua GP, Tan HK, Gandhi M. Information sources and online information seeking behaviours of cancer patients in Singapore. Ecancermedicalscience 2018;12:880.

26. Wu C, Wu Z, Yang L, et al. Evaluation of the clinical outcomes of telehealth for managing diabetes: A PRISMA-compliant meta-analysis. Medicine (Baltimore) 2018;97:e12962.

27. Jeminiwa R, Hohmann L, Qian J, et al. Impact of eHealth on medication adherence among patients with asthma: A systematic review and meta-analysis. Respir Med 2019;149:59-68.

28. Jiang J, Zhu Q, Zheng Y, et al. Perceptions and Acceptance of mHealth in Patients With Cardiovascular Diseases: A Cross-Sectional Study. JMIR Mhealth Uhealth 2019;7:e10117.

29. Dou K, Yu P, Deng N, et al. Patients' Acceptance of Smartphone Health Technology for Chronic Disease Management: A Theoretical Model and Empirical Test. JMIR Mhealth Uhealth 2017;5:e177.

30. Van Velthoven MH, Cordon C. Sustainable Adoption of Digital Health Innovations: Perspectives From a Stakeholder Workshop. J Med Internet Res 2019;21:e11922.

31. Ministry of Health (Singapore). MOH Launches First Regulatory Sandbox To Support Development Of Telemedicine [press release]. Available online: https:// www.moh.gov.sg/news-highlights/details/moh-launchesfirst-regulatory-sandbox-to-support-development-oftelemedicine. Published: April 18, 2018. Accessed: January 6, 2019.

doi: $10.21037 /$ mhealth.2019.08.07

Cite this article as: Hossain I, Ang YN, Chng HT, Wong PS. Patients' attitudes towards mobile health in Singapore: a crosssectional study. mHealth 2019;5:34. 